ethane, and the spectrum was recorded with a relatively low resolution. A direct comparison between this spectrum and that reported by us is therefore rather difficult.

In view of the findings described by Ward, it is not impossible, however, to still assign our spectrum to negative ions derived from $s$-trinitrobenzene. The peculiar single nitrogen interaction of our system in which no alkali metals were present, is perhaps connected with some kind of interaction with the tetrahydrofuran molecules. It may be suggested that the complex between tetrahydrofuran and $s$-trinitro benzene involves three molecules of tetrahydrofuran and one molecule of $s$-trinitrobenzene according to the formula given below. The presence of three simultaneous spin systems each enclosing the aromatic nucleus with the three protons and one nitro-group with the attached tetrahydrofuran molecule, could possibly explain the obtained spectrum.<smiles>O=[N+]([O-])c1cc([N+](=O)[O-])c([C@H](OC(F)F)C(F)F)c([N+](=O)[O-])c1</smiles>

1. Lagercrantz, C. and Yhland, M. Acta Chem. Scand. 16 (1962) 1043.

2. Ward, R. L. J.Am. Chem. Soc. 83 (1961) 1296.

3. Maki, A. H. and Geske, D. H. J. Chem. Phys. 33 (1960) 825.

Received July 31, 1962.

\section{Interconversion of 1-Phenyl-} 5-mercapto-1,2,3-triazole and 5-Phenylamino-1,2,3-thiadiazole

T. KIN D T - L A R S E N and

CHRISTIAN PEDERGEN

Department of Organic Chemistry, University of Technology, Copenhagen, Denmark

From the reaction between 1-phenyl-5chloro-1,2,3-triazole (I) and sodium hydrogen sulphide one would expect to get 1-phenyl-5-mercapto-1,2,3-triazole (II).
Hess has, however, shown ${ }^{1}$ that this reaction produces a mixture of two compounds one of which is a sulphide with the structure (VIII). The other product, with melting point $173^{\circ}$, has the composition corresponding to (II) but since it shows no acidic properties it cannot be a mercaptotriazole and Hess proposed that it has the structure (III). Hess, furthermore, found that this compound would dissolve in hot, aqueous sodium hydroxide; acidification of the alkaline solution gave a new, isomeric compound with m.p. $65^{\circ}$ for which the structure (II) was proposed. The lower melting isomer, when heated in ligroin, rearranged to the higher melting isomer.

Since the structures (II) and (III) are tautomeric it is unlikely that they should represent two different compounds and it has now been shown that the higher melting isomer is identical with the known 5-phenylamino-1,2,3-thiadiazole (IV) which is easily prepared from phenyl isothiocyanate and diazomethane ${ }^{2}$.

The lower melting isomer was prepared from authentic 5-phenylamino-1,2,3-thiadiazole (IV) by heating with sodium hydroxide according to Hess ${ }^{1}$. That it is 1-phenyl-5-mercapto-1,2,3-triazole (II), as proposed by Hess, was shown by convert.
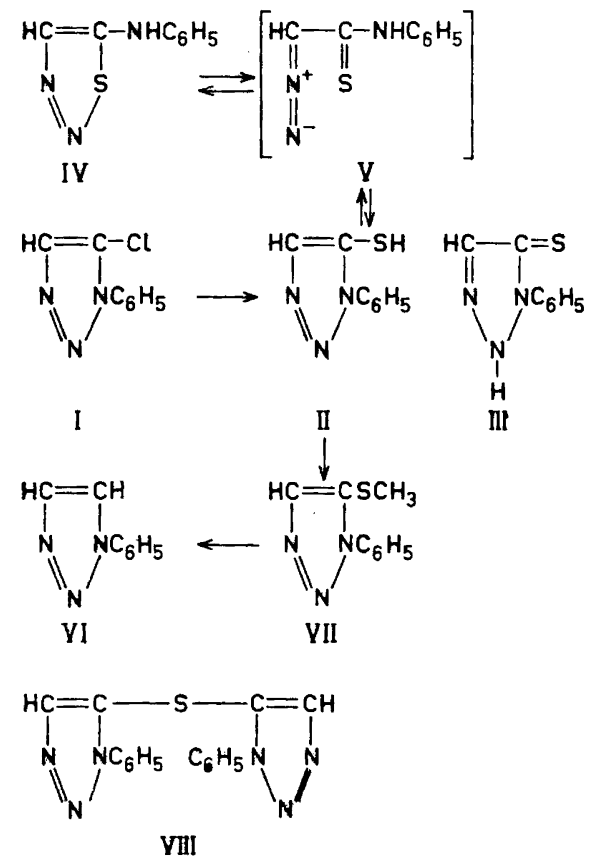

Acta Chem. Scand. 16 (1962) No. 7 
ing it to 1-phenyl-5-methylmercapto-1,2,3triazole (VII) which, when desulphurized with Raney nickel, gave the known 1-phenyl-1,2,3-triazole (VI). The direct desulphurization of (II) was not attempted because of the instability of this compound.

The primary product in the reaction of 1-phenyl-5-chloro-1,2,3-triazole (I) and sodium hydrogen sulphide is probably the mercaptotriazole (II) which then, under the conditions of the reaction, rearranges into (IV). The interconversion of (II) and (IV) is probably taking place via the diazothioacetic anilide (V) in analogy with the rear. rangement between 1-phenyl-5-amino1,2,3-triazole and 5-phenylamino-1,2,3 triazole where a diazocompound similar to (V) is assumed to be an intermediate ${ }^{3}$. Sheehan and Izzo ${ }^{2}$ assumed a tautomeric form of (V) to be an intermediate in the formation of 5-phenylamino-1,2,3-thiadiazole from phenyl isothiocyanate and diazomethane.

Experimental. Reaction of 1-phenyl-5-chloro1,2,3-triazole with sodium hydrogen sulphide. To a solution of 1-phenyl-5-chloro-1,2,3triazole ${ }^{4,5}(2.5 \mathrm{~g})$ in ethanol $(35 \mathrm{ml})$ was added $7.5 \mathrm{ml}$ of $22 \%$ aqueous sodium hydrogen sulphide (prepared by saturating a solution of sodium hydroxide $(8.0 \mathrm{~g})$ in water $(37 \mathrm{ml})$ with hydrogen sulphide at room temperature). The solution was kept at room temperature for 7 days during which time a crystalline precipitate formed; it was filtered off, washed with water and ethanol and dried. Yield $0.70 \mathrm{~g}$ $(28 \%)$, m.p. $168-170^{\circ}$. After two recrystallizations from ethanol the compound melted at $172-173^{\circ}$. (Found: C 53.95; H 4.00; N 23.80. Calc. for $\mathrm{C}_{8} \mathrm{H}_{7} \mathrm{~N}_{3} \mathrm{~S}$ : C 54.19; $\mathrm{H} \mathrm{3.99;} \mathrm{N} 23.72$ ). The product was identical in infra red spectrum and mixed melting point with 5-phenylamino1,2,3-thiadizole (IV) prepared according to Sheehan ${ }^{2}$. When the reaction was performed at $100^{\circ}$ in a sealed tube the only product that could be isolated was the sulphide (VIII).

1-Phenyl-5-mercapto-1,2,3-triazole. $4.0 \mathrm{~g}$ of 5-phenylamino-1,2,3-thiadiazole was added to a solution of sodium hydroxide $(1.0 \mathrm{~g})$ in water $(25 \mathrm{ml})$. The mixture was heated to $c a$. $90^{\circ}$ for a few minutes until the thiadiazole was dissolved, and the slightly coloured solution was filtered through activated carbon. The filtrate was cooled in ice; when acidified with dilute hydrochloric acid an oil was precipitated which crystallized on scratching. The product was filtered off and washed with water. Yield $3.30 \mathrm{~g}(82 \%)$, m.p. $63-65^{\circ}$. Two recrystalliza- tions from ether-pentane gave pure 1-phenyl5-mercapto-1,2,3-triazole with m.p. 64-65 (Hess ${ }^{1}$ reported m.p. $6^{\circ}$ ). (Found: C 54.35; $\mathrm{H} 4.02 ; \mathrm{N} 23.86 ; \mathrm{S}$ 18.03. Calc. for $\mathrm{C}_{8} \mathrm{H}_{7} \mathrm{~N}_{8} \mathrm{~S}$ : C 54.19; H 3.99; $\mathrm{N}$ 23.72; $\mathrm{S}$ 18.08). The compound was decomposed when kept at room temperature for a fow weeks.

1-Phenyl-5-methylmercapto-1,2,3-triazole. 5Phenylamino-1,2,3-thiadiazole $(10.0 \mathrm{~g})$ was suspended in $150 \mathrm{ml}$ of $1 \mathrm{~N}$ sodium hydroxide and heated to $c a .90^{\circ}$ for a few minutes until it was dissolved. The solution was filtered through activated carbon, cooled and stirred with methyl iodide $(20 \mathrm{ml})$ for $2 \mathrm{~h}$ at room temperature. Excess methyl iodide was removed by blowing air through the mixture and the product was then extracted with ether. The ether solution was washed with water and dried with magnesium sulphate. The ether was removed in vacuo and the residue was distilled yielding $6.6 \mathrm{~g}(61 \%)$ of 1-phenyl-5-methylmercapto-1,2,3-triazole as a yellow oil with b.p. $140^{\circ}(0.5 \mathrm{~mm})$. (Found: C $56.50 ; \mathrm{H} 4.88$; $\mathrm{N} 22.80 ; \mathrm{S}$ 16.88. Calc. for $\mathrm{C}_{9} \mathrm{H}_{9} \mathrm{~N}_{3} \mathrm{~S}$ : C 56.50; H 4.75; N 22.00; S 16.76).

1-Phenyl-1,2,3-triazole. To a solution of 1 phenyl-5-methylmercapto-1,2,3-triazole (1.0 g) in ethanol $(20 \mathrm{ml})$ was added $c a .5 \mathrm{~g}$ of Raney nickel and the mixture was heated on the steam bath for $8 \mathrm{~h}$. The Raney nickel was filtered off and washed twice with hot ethanol and the ethanol was removed in vacuo at room temperature. The residue was dissolved in ether and treated with activated carbon. Addition of pentane precipitated $430 \mathrm{mg}(56 \%)$ of 1-phenyl-1,2,3-triazole with m.p. $54-55^{\circ}$ (Dimroth ${ }^{6}$ recorded $55-56^{\circ}$ ). Mixed melting point and infrared spectrum proved that the product was identical with an authentic sample.

Microanalysis were made by $\mathrm{Mr}$. Preben Hansen.

This work is part of an investigation supported by Kai Hansen's Fond.

1. Hess, F. Diss., München 1909.

2. Sheehan, J. C. and Izzo, P. T. J. Am. Chem. Soc. 71 (1949) 4059.

3. Lieber, E., Ramachandra Rao, C. N. and Chao, T. S. J. Am. Chem. Soc. 79 (1957) 5962.

4. Dimroth, O. Ann. 364 (1909) 203.

5. Lieber, E., Chao, T. S. and Ramachandra Rao, C. N. J. Org. Chem. 22 (1957) 654.

6. Dimroth, O. Ber. 35 (1902) 1029.

Received July 29, 1962. 